\title{
DNA Detection by SERS: Hybridisation Parameters and the Potential for Asymmetric PCR
}

Received 00th January 20xx, Accepted 00th January 20xx DOI: $10.1039 / x 0 x \times 00000 x$

\author{
Daniel Macdonald, ${ }^{a}$ Ewen Smith, ${ }^{a}$ Karen Faulds ${ }^{a}$ and Duncan Graham ${ }^{a}$
}

The use of surface enhanced Raman scattering (SERS) for the detection of DNA has significant potential in terms of sensitivity, multiplex target detection and robust signal detection from nanoparticles. Current methods are usually performed with short chain DNA fragments or require additional separation steps for detection of longer chain fragments. We present an integrated method for the rapid and sensitive detection of extended ( $\geq 100$-base) nucleic acids with reduced preparation and sample separation steps. Key to this is the DNA sequence-specific assembly of silver nanoparticles labelled with a Raman tag to provide an enhanced signal from the tag and hence molecular recognition of the target DNA. DNA probe orientation and hybridisation procedures are critical for the success of this assay. Effective detection of extended nucleic acids was achieved with head-to-head probes and by adding polyethylene glycol 10000 (PEG 10000) to the hybridisation buffer. This gave a 34-fold discriminatory enhancement factor when applied to a synthetic target. A structured approach toward maximising hybridisation procedures and SERS response is described, followed by an initial demonstration of SERS detection of single-stranded DNA target amplified by asymmetric PCR which was used without further separation. This has implications for future developments in using SERS for DNA detection due to the new-found ability to integrate SERS with asymmetric PCR.

\section{Introduction}

Surface Enhanced Raman Spectroscopy (SERS) is a powerful vibrational spectroscopy technique providing Raman signal intensities that are orders of magnitude above that of Raman scattering alone. SERS provides significant multiplexing potential due to narrow spectral features corresponding to unique molecular vibrational modes and has been widely applied for biomolecular analysis of nucleic acids, proteins and lipids using an extensive range of methodologies. ${ }^{1,} 2$ We previously published the first report of a suspension-based SERS methodology for assembly of DNA functionalised and dyecoded silver nanoparticles (AgNPs) using short-chain, singlestranded DNA (ssDNA) targets. ${ }^{3}$ This emphasised the key principle that a SERS response may be produced in solution, founded entirely upon sequence-specific base-pairing. DNA functionalised AgNPs captured Raman-tags within hotspots upon target hybridisation, generating a SERS signal with multiplexing potential.

However, detection of unamplified genomic DNA presents further challenges including: (a) a low target concentration, (b) genetic sequences thousands of bases in length (c) a doublestranded DNA (dsDNA) structure preventing direct hybridisation of DNA functionalised nanoparticles. Therefore, nucleic acid

\footnotetext{
a. Centre for Molecular Nanometrology, Department of Pure and Applied Chemistry, WestCHEM, University of Strathclyde, Technology and Innovation Centre, 99 George Street, Glasgow, G1 1RD, UK.

+ Electronic Supplementary Information (ESI) available: Additional UV-Vis extinction spectra, SERS spectra, conjugate probe sequences, target sequences and PCR primer sequences. See DOI: 10.1039/x0xx00000x
}

detection is typically preceded by target amplification, thereby significantly increasing the concentration of detectable longchain dsDNA fragments with discriminatory value. Currently, dsDNA amplification products require increasingly complex, indirect SERS detection methodologies as compared to the short, synthetic SSDNA targets that are commonly utilised for the assembly of nanoparticles.

To address the issue of detecting long-chain dsDNA amplification products, we reported a PCR-SERS method for detection of mutations in the cystic fibrosis trans-membrane conductance regulator (CFTR) gene. ${ }^{4}$ Dye-labelled primers were incorporated within the amplification product and discriminatory value was provided by a multi-step process requiring annealing of biotinylated probes, streptavidin-coated magnetic bead separation, elution of PCR product and spermine-mediated aggregation of nanoparticles to "switch on" SERS. We further demonstrated the multiplexing potential with the detection of six human papilloma virus genotypes. ${ }^{5}$ In this instance, the primers were biotinylated, and the specificity was conferred by dye-labelled probes. Li et al., have applied this PCR-SERS method for multiplex detection of mutations associated with lung cancer, colorectal cancer and thalassemia respectively, substituting magnetic bead separation for PCR purification.6, 7, 8 Furthermore, Wee et al., reported a PCR-SERS method for multiplex detection of melanoma associated mutations in circulating tumour DNA, omitting sperminemediated aggregation in favour of enrichment and SERS detection directly on the surface of magnetic beads. ${ }^{9}$ This was achieved using a biotinylated reverse primer for magnetic bead capture, and a forward primer modified with an internal spacer 
and 5' overhang for direct hybridisation to dye-coded and DNA functionalised nanoparticles.

Current PCR-SERS methods are highly reliant upon physical separation by magnetic bead separation or spin-column purification, which are effective but time-consuming and costly due to covalent modification of primer or probe sequences. Therefore, we have focused on the detection of long-chain fragments reminiscent of amplification products, with the aim to directly assemble nanoparticles without requirement for primer or probe modification and physical separation. Optimised conditions for the rapid detection of long-chain DNA fragments are described, maximising SERS enhancement based upon key criteria. These include the addition of high-molecular weight polyethylene glycol as a molecular crowding agent to induce rapid hybridisation to long-chain nucleic acid fragments, and maximisation of nanoparticle proximity in a head-to-head orientation for optimal SERS enhancement. The proof-ofprinciple detection methodology was achieved using a 100-base synthetic target reminiscent of amplification products. ssDNA was then amplified from a biological template by asymmetric PCR, avoiding pre-analytical steps used to isolate ssDNA such as magnetic bead separation or enzymatic digestion and the product detected directly by SERS analysis.

\section{Experimental}

\section{Nanoparticle Synthesis}

Silver nanoparticles (AgNPs) were synthesised using the method published by Leopold and Lendl with reagent quantities adjusted for a $300 \mathrm{~mL}$ batch volume. ${ }^{23}$ Concentrations for each reagent were as follows; hydroxylamine hydrochloride (1.5 $\mathrm{mM})$, sodium hydroxide $(3 \mathrm{mM})$, silver nitrate $(10 \mathrm{mM})$. Hydroxylamine hydrochloride and sodium hydroxide aliquots were dissolved in a conical flask containing $270 \mathrm{~mL}$ of MQ water. Silver nitrate was dissolved separately in $30 \mathrm{~mL}$ of $\mathrm{MQ}$ water, then added to the reaction mixture and left to stir vigorously for $15 \mathrm{~min}$ at room temperature.

\section{Oligonucleotide Probe and Synthetic Target Design and Preparation}

A 100 base synthetic target sequence ( $47 \%$ GC content) was selected from the 18S rRNA gene sequence for Candida krusei (also referred to as Pichia kudriavzevii), as identified by the GenBank accession number KP202859.1. Probes were evaluated in silico using the OligoAnalyzer 3.1 web application. ${ }^{24}$ Probes were designed based upon criteria to minimise secondary structures. Hairpin, self-dimer and heterodimer structures were identified and accounted for by maintaining a minimum delta $G$ threshold $\geq-9$ units thus reducing the likelihood of secondary structure formation. HPLC purified oligos were ordered from ATDBio (Southampton, UK). Probe sequences were modified with $3 \times$ hexaethylene glycol (HEG) units and a terminal thiol. Lyophilised oligos were briefly centrifuged at $3300 \mathrm{rcf}$ and resuspended in $\mathrm{MQ}$ water to a concentration of $100 \mu \mathrm{M}$. All probe and target sequences are contained within Table S1.

Buffers
$0.1 \mathrm{M}(10 \mathrm{mM}$ phosphate and $0.1 \mathrm{M} \mathrm{NaCl})$ and $0.3 \mathrm{M}(10 \mathrm{mM}$ phosphate and $0.3 \mathrm{M} \mathrm{NaCl}$ ) phosphate buffered saline (PBS) were prepared and adjusted to $\mathrm{pH} 7.4$ and filtered prior to use. $250 \mathrm{mM}$ citrate buffer ( $250 \mathrm{mM}$ trisodium citrate) was prepared and adjusted to $\mathrm{pH} 3.0$.

\section{Conjugate Preparation}

Nanoparticle concentrations were approximated using the Beer-Lambert law $(A=\varepsilon \times I \times C$ where $A$ is absorbance, $\varepsilon$ is the extinction coefficient, $I$ is the pathway length and $C$ is the concentration) and extinction coefficient $537 \times 10^{8} \mathrm{M}^{-1} \mathrm{~cm}^{-1}$ for $50 \mathrm{~nm}$ nanoparticles. ${ }^{25}$ Extinction spectra were gathered using a Varian Cary 300 Bio UV-Vis Spectrophotometer (Agilent, California, USA). Conjugates were prepared using a modified $\mathrm{pH}$ method as published by Zhang et al. ${ }^{26}$ Nanoparticle stock was dispensed in $900 \mu \mathrm{L}$ volumes followed by the addition of oligo probes at a 7500: 1 oligo to nanoparticle molar ratio. Samples were vortexed and left to equilibrate for $1 \mathrm{~h}$, followed by two stepwise additions of $250 \mathrm{mM}$ citrate buffer (pH 3.0). Initially 30 $\mu \mathrm{L}$ of citrate buffer was added and shaken for 15 minutes, followed by a further $30 \mu \mathrm{L}$ addition. The conjugate was completed with addition of MQ water to a total volume of $1 \mathrm{ml}$ and left to equilibrate for 45 minutes without shaking. Following probe immobilisation, conjugates were centrifuged at 3300 rcf for $20 \mathrm{~min}$ and the supernatant removed. The conjugate was then resuspended in $500 \mu \mathrm{L}$ of $0.1 \mathrm{M}$ PBS $(\mathrm{pH}$ 7.4) for a $2 \mathrm{x}$ working stock. Addition of a Raman-tag was required for one of the conjugate probe sequences. The dye-coded conjugate was resuspended in $1 \mathrm{ml} \mathrm{MQ}$ water rather than $\mathrm{PBS}$, and the concentration calculated as previously. Malachite green isothiocyanate (MGITC) was added from a $100 \mu \mathrm{M}$ stock solution at a 2500: 1 molar ratio and left to sonicate for 3 minutes. The conjugate was then centrifuged at $3300 \mathrm{rcf}$ for 20 minutes, the supernatant removed, and resuspended in $500 \mu \mathrm{L}$ of $0.1 \mathrm{M}$ PBS ( $\mathrm{pH} 7.4$ ) for a $2 x$ working stock.

\section{Polymerase Chain Reaction}

A 383 base biological target sequence ( $45 \%$ GC content) was selected for amplification from the $18 \mathrm{~S}$ rRNA gene sequence for Candida krusei, as identified by the GenBank accession number KP202859.1. PCR reactions were performed on a Stratagene Mx3005P thermocycler (Agilent, California, USA) using reagents supplied by New England Biolabs (Hitchin, UK). Reactions were performed in $50 \mu \mathrm{L}$ volumes. The mastermix consisted of; $1 \mathrm{x}$ PCR buffer, $0.8 \mathrm{mM}$ dNTPs, $0.8 \mu \mathrm{M}$ forward (excess) primer, $0.08 \mu \mathrm{M}$ reverse (limiting) primer, $2.5 \mathrm{mM}$ magnesium chloride and 2.5 $\mathrm{U}$ Hot Start Taq DNA Polymerase. The mastermix was spiked with $1 \mu \mathrm{L}$ of Candida krusei plasmid DNA, and a total volume of $50 \mu \mathrm{L}$ was made up with addition of $\mathrm{MQ}$ water. Prior to optimisation of the described asymmetric protocol, standard PCR was implemented with an equal primer concentration of $0.8 \mu \mathrm{M}$. The reverse primer was 5' phosphate modified to allow for exonuclease digestion and size comparison to the asymmetric ssDNA amplicon. Primer sequences can be found in the supplementary materials. PCR was performed with $45 x$ cycles of; melting at $94^{\circ} \mathrm{C}$ for 30 seconds, annealing at $57^{\circ} \mathrm{C}$ for 30 seconds and elongation at $72{ }^{\circ} \mathrm{C}$ for 30 seconds and a final elongation cycle at $72{ }^{\circ} \mathrm{C}$ for 7 minutes.

Lambda Exonuclease Treatment of PCR Product 
Lambda exonuclease (Fisher Scientific UK Ltd, Loughborough, UK) was used to digest PCR product into ssDNA form by following of manufacturer instructions. Enzymatic digestion was performed in a $50 \mu \mathrm{L}$ reaction volume. $10 \mathrm{U}$ of lambda exonuclease was added for every $2 \mu \mathrm{g}$ of DNA added to the reaction mix, and left to digest at $37^{\circ} \mathrm{C}$ for $30 \mathrm{~min}$. The digestion was then stopped by heating at $80^{\circ} \mathrm{C}$ for $10 \mathrm{~min}$.

\section{Gel Electrophoresis}

Gel electrophoresis was performed to confirm DNA molecular weight and PCR products were size separated at $160 \mathrm{~V}$ for 30 $\min$ in $2 \%$ agarose gel $(\mathrm{w} / \mathrm{v})$ pre-stained with $1 \times$ GelRed $^{\mathrm{TM}}$ dye (Biotium, Hayward, USA). Sizes were referenced against a 50 bp DirectLoad ${ }^{\mathrm{TM}}$ DNA ladder (Sigma-Aldrich, Poole, UK).

\section{Hybridisation Assay}

Conjugates were hybridised in Eppendorf tubes containing 0.3 $M$ PBS with addition of PEG $(\mathrm{w} / \mathrm{v})$ and DMSO $(\mathrm{v} / \mathrm{v})$ at the required concentrations. Eppendorfs were placed in a preheated heat-block prior to addition of reagents. The final volume for each sample of hybridisation reaction mix was 250 $\mu \mathrm{L}$ per replicate. Buffer and additives were initially aliquoted, followed by addition of each conjugate for a final concentration of $10 \mathrm{pM}$. The synthetic target sequence was aliquoted from a $1 \mu \mathrm{M}$ working stock for a final concentration of $10 \mathrm{nM}$. PCR target was added in a volumetric approach. Samples were briefly removed from the heat-block, vortexed and replaced within the heat-block prior to hybridisation. Hybridisation was implemented for $1 \mathrm{~h}$ followed by SERS analysis. The annealing temperature was set at $45{ }^{\circ} \mathrm{C}$ for the synthetic target hybridisation, and $95{ }^{\circ} \mathrm{C}$ followed by gradual cooling to $55{ }^{\circ} \mathrm{C}$ (within $1 \mathrm{~h}$ ) for the PCR target hybridisation.

\section{SERS Analysis}

SERS was performed on a Snowy Range Instrument - Sierra 2.0 Raman Spectrometer (Wyoming, USA) at $532 \mathrm{~nm}$ wavelength using $10 \%(5 \mathrm{~mW})$ laser power. This was performed in a black 96-well plate with $250 \mu \mathrm{L}$ of conjugate hybridisation mix aliquoted into each well. Optimal plate distancing was calibrated using an absolute ethanol standard placed on an adjustable platform. Spectra were gathered in $5 \mathrm{x}$ accumulations per replicate and averaged. The integration time was adjusted in accordance with sample signal intensity Spectral data was baseline corrected using the SpectraGryph optical spectroscopy software, version 1.0. ${ }^{27}$ Each spectrum was individually corrected using default adaptive baseline correction settings.

\section{Results and Discussion}

The basic premise of the methodology presented is founded upon sequence-specific assembly of AgNPs upon addition of a complementary DNA target. When a complementary sequence is added, AgNPs are cross-linked in proximity. This may be observed by eye as a colour change or measured as a shift in the extinction spectra due to localised surface plasmon resonance coupling. The junctions between nanoparticles positioned in proximity amplify electromagnetic radiation in the near field and these regions are referred to as "hotspots". Molecules present within these regions may display surface-enhanced Raman cross-sections. DNA has an intrinsically weak Raman cross-section and does not readily provide sequence-specific information. Therefore, we have immobilised malachite green isothiocyanate (MGITC) upon the surface of the nanoparticles corresponding to one of the two probe sequences used for detection. Raman reporters have strong Raman cross-sections and combinations of reporters may be selected for multiple probe sequences providing the capacity for multiplex detection of nucleic acids. If no target is present, the Raman reporter signal is not enhanced and if the target is present, the signal is enhanced upon nanoparticle assembly as the Raman reporter is positioned within the hotspot regions. The discriminatory value may be calculated by comparing the spectral peak intensity of target-assembled and non-assembled samples, providing a SERS on-to-off discriminatory ratio. The magnitude of SERS enhancement is particularly sensitive to interparticle distancing, and therefore the investigation began with the identification of optimal probe orientations. The conjugate components and principles of the head-to-head, head-to-tail and tail-to-tail orientations investigated are displayed in Figure 1.

A proof-of-principle investigation was implemented to confirm the potential for the detection of long-chain nucleic acid fragments ( $\geq 100$ bases) using hybridisation probes and a complementary target sequence. A 100-base synthetic DNA target based upon the Candida krusei 18S rRNA gene was selected. This was within the typical size range of amplified DNA fragments and was selected based upon minimal capacity for secondary structure formation. The probe hybridisation region

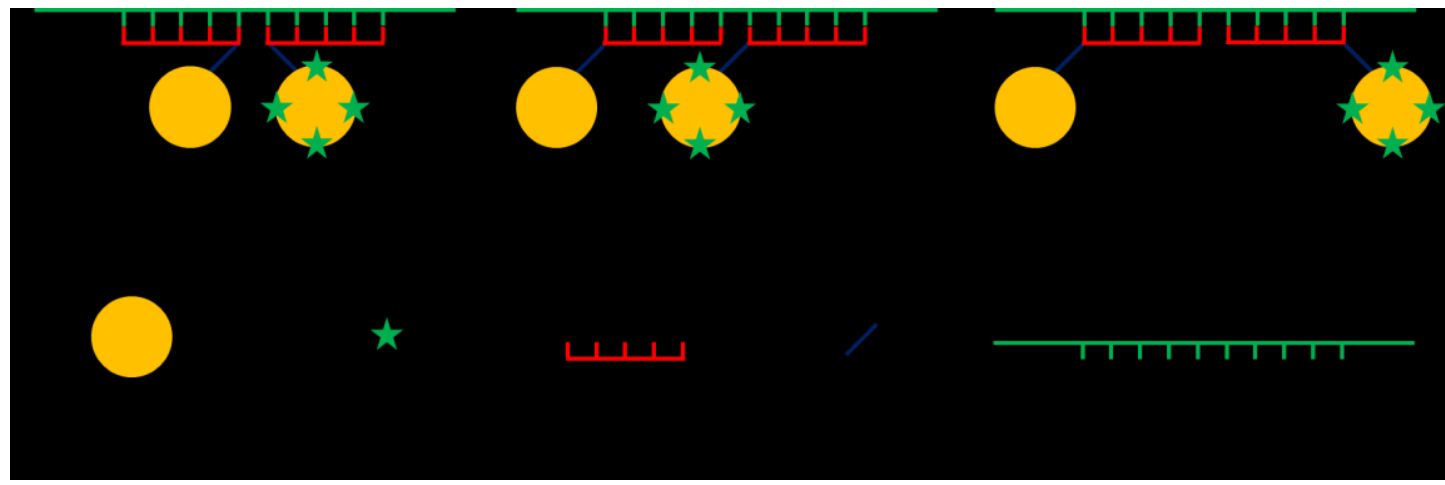

Figure 1. Schematic depicting hybridisation probe orientations: A. Head-to-head orientation B. Head-to-tail orientation C. Tail-to-tail orientation. Conjugate composition detailed underneath. MGITC Raman-tag added to one probe conjugate only. 
was checked for species-specificity by BLAST sequence analysis. The nanoparticle proximity and relation to SERS signal was investigated by comparison of the head-to-head, head-to-tail and tail-to-tail orientations. Prior investigation by Mabbott et al. described poor enhancement in the tail-to-tail orientation due to interparticle separation reducing the plasmonic coupling proximity required for SERS enhancement within hotspot regions. ${ }^{10}$ Conversely, a head-to-head orientation provided the closest proximity, but was limited by steric hindrance. A headto-tail orientation provided optimal SERS discrimination as the nanoparticles were proximal enough for a SERS response, whilst distant enough to avoid steric hindrance.

We observed that the addition of high molecular weight polyethylene glycol (PEG) to the $0.3 \mathrm{M}$ PBS buffer instigated hybridisation in each orientation including the head-to-head orientation. PEG was selected as a molecular crowding reagent due to commercial availability, low cost, variable molecular weight, non-ionic properties and biologically inert status. PEG has been identified as a hybridisation enhancer and did not cause loss of colloidal suspension upon assembly providing an extended timeframe for analysis. ${ }^{11}$ The improved hybridisation observed is likely due to multiple mechanisms including volume exclusion, decreased molecular diffusion, altered solvent dielectric constants and changes to biomolecular equilibrium constants. ${ }^{12,13}$ PEG may also condense and compact DNA in coilglobule transitions. ${ }^{14,}{ }^{15}$ Additionally, PEG can destabilise or stabilise DNA duplexes upon addition of low or high molecular weight PEG respectively. ${ }^{16}$ Initially, PEG 6000 was selected as the reagent of choice. Hybridisation was observable by UV-Vis spectroscopy with a decrease in the extinction peak and emergence of a shoulder at approximately $500 \mathrm{~nm}$ for the headto-head orientation (Figure 2).

PEG 6000 was added at an optimal concentration of $5 \%$ $(\mathrm{w} / \mathrm{v})$ to induce rapid hybridisation. Hybridisation kinetics were reduced at lower concentrations $(3 \%)$ and slight non-specific aggregation occurred at higher concentrations $(7 \%)$, as indicated by a slight decrease in the maximum extinction peak and increase at longer wavelengths ranging from $500 \mathrm{~nm}-800$ $\mathrm{nm}$ (Figures S1-S3). Hybridisations were implemented at $45{ }^{\circ} \mathrm{C}$. This was required to prevent non-specific dimerization of the probes in the buffer containing the PEG crowding agent.

The relation between the SERS enhancement and probe orientations was investigated, and the SERS discriminatory onto-off ratios were calculated upon comparison of the MGITC peak intensity at $1618 \mathrm{~cm}^{-1}$ in samples containing complementary and non-complementary DNA respectively. The discriminatory value correlated in agreement with the proximity of probe orientations (Figures S4-S6). The tail-to-tail orientation did not generate SERS enhancement or discrimination with a 1: 1 on-to-off ratio. Hybridisation in the head-to-tail and head-to-head ratios displayed improved SERS enhancement and discrimination with on-to-off ratios of 6: 1 and 13: 1 respectively.

The effect of PEG molecular weight was investigated in the head-to-head orientation. PEG 2000, PEG 6000 and PEG 10000 were compared using a standard $5 \%(\mathrm{w} / \mathrm{v})$ concentration for identification of optimal molecular weight relating to SERS

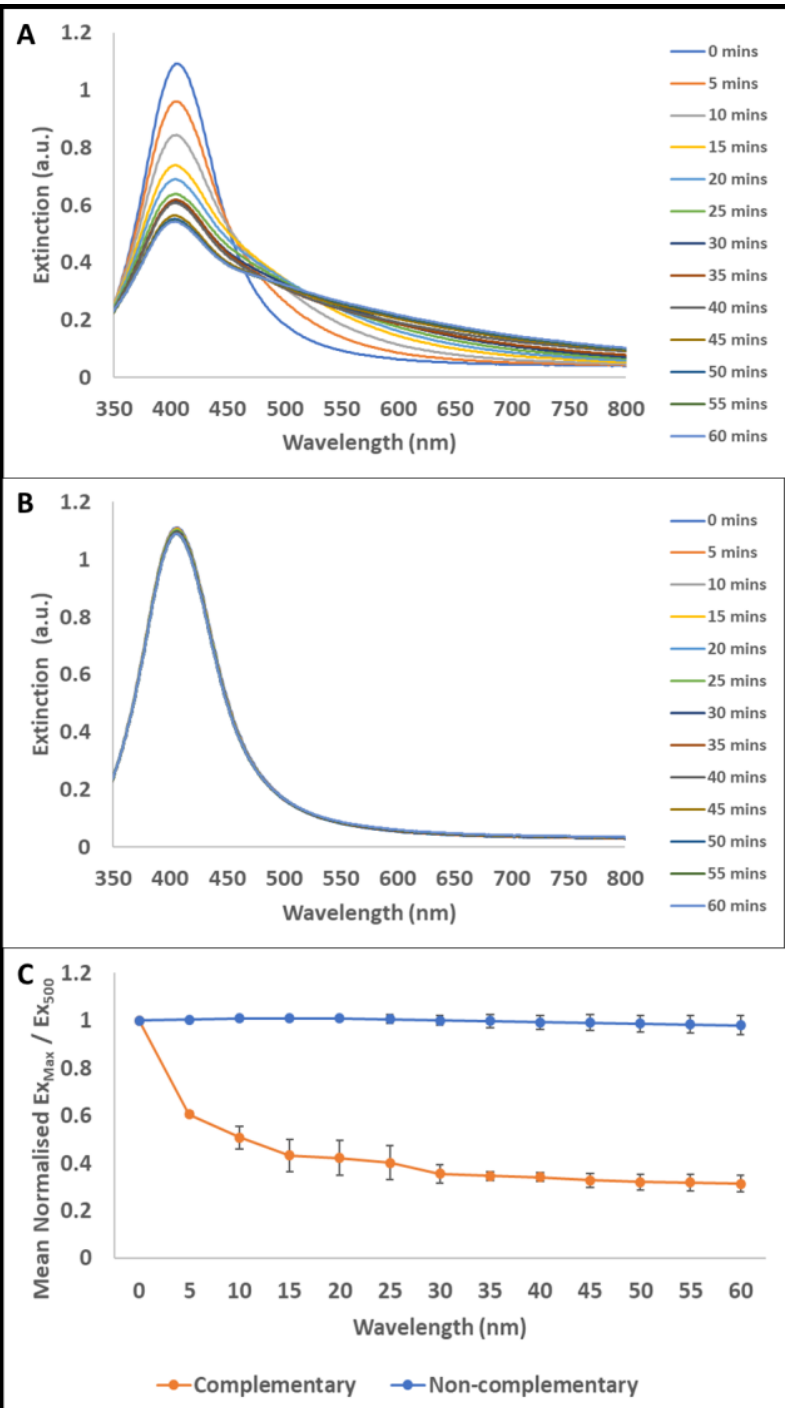

Figure 2. UV-Vis extinction kinetics: Conjugates (10 pM) hybridised to synthetic target $(10 \mathrm{nM})$ in the head-to-head orientation using 0.3 M PBS with $5 \%$ PEG 6000 at $45^{\circ} \mathrm{C}$ for 1 hour. A. Addition of 100-base complementary target. B. Addition of a 100-base noncomplementary sequence. C. Mean $(\mathrm{N}=3)$ normalised ratio of maximum extinction $(\mathrm{nm}) / 500 \mathrm{~nm}$ extinction values at 5-minute intervals for both complementary and non-complementary DNA with error bars representative of standard deviation. Maximum extinction / $500 \mathrm{~nm}$ data normalised to extinction maximum at 0 minutes.

discrimination and intensity (Figure S7-S9). Addition of PEG 2000 displayed high SERS intensity with accumulations at 0.05 seconds, but poor discrimination with an on-to-off ratio of 2: 1 . Addition of PEG 6000 improved discriminatory value with an onto-off ratio of 12: 1, although the SERS intensity was weaker, and a longer accumulation time of 0.1 seconds was applied. Addition of PEG 10000 provided significant discrimination with an on-to-off ratio of 8: 1 whilst generating high SERS intensities using a short accumulation time of 0.05 seconds. The $5 \%$ PEG 10000 hybridisation buffer was further optimised with Tween 20 to reduce non-specific dimerization of the probes in the buffer containing the PEG crowding agent. At concentrations of $0.1 \%$ and $0.05 \%(\mathrm{v} / \mathrm{v})$, Tween 20 significantly improved 


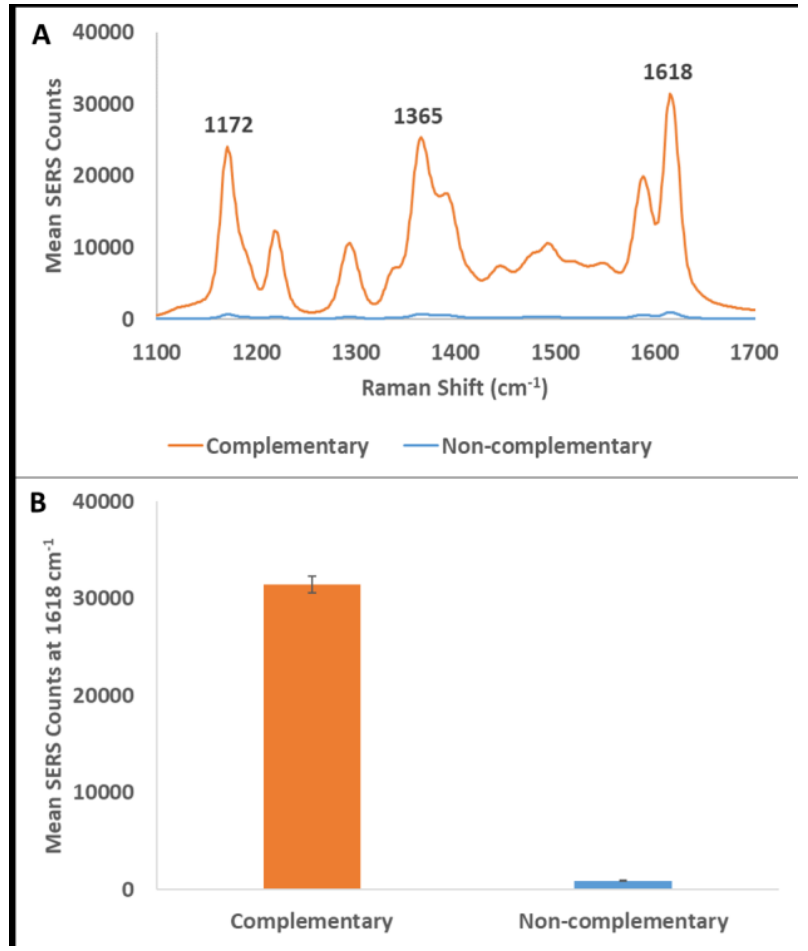

Figure 3. SERS analysis using optimised conditions: Conjugates (10 pM) hybridised to synthetic target $(10 \mathrm{nM})$ in head-to-head orientation using $0.3 \mathrm{M}$ PBS with $5 \%$ PEG 10000 and $0.05 \%$ Tween 20 at $45{ }^{\circ} \mathrm{C}$ for 1 hour. A. Mean ( $\left.\mathrm{N}=3\right)$ SERS spectra $(0.05 \mathrm{~s}$ accumulation time). B. On-to-off ratio of 34: 1 with error bars representative of standard deviation. Data accumulated using a 532 $\mathrm{nm}$ laser wavelength and $10 \%(5 \mathrm{~mW})$ laser power.

discriminatory value with on-to-off ratios of 28: 1 (Figure S10) and 34: 1 respectively (Figure 3).

The proof-of-principle detection methodology concluded upon the detection of an extended 100-base synthetic DNA sequence, with optimal hybridisation occurring in $1 \mathrm{~h}$ at $45^{\circ} \mathrm{C}$ using 0.3 M PBS buffer containing $5 \%(w / v)$ PEG 10000 and 0.05 $\%(\mathrm{v} / \mathrm{v})$ Tween 20 . The on-to-off discriminatory ratio of 34: 1 achieved using these conditions was a significant improvement upon our prior achievement for which an approximate on-to-off ratio of 3: 1 was observed for detection of a synthetic Candida krusei target. ${ }^{10}$ This represented a ten-fold improvement in discriminatory value.

Detection of a biologically relevant target amplicon was then attempted without pre-analytical purification, separation or wash steps. For the direct assembly of nanoparticles, a ssDNA target is required. We applied asymmetric PCR to amplify a 383base sSDNA fragment of the Candida krusei 18S rRNA gene in a one-pot reaction based upon linear-after-the-exponential (LATE-PCR) experimental design criteria. ${ }^{17,}{ }^{18}$ Asymmetric PCR requires a primer concentration imbalance, with one of two primers present in excess to that of the limiting primer. Exponential amplification of dsDNA occurs until the limited primer is exhausted and linear amplification then proceeds to synthesise ssDNA from the dsDNA pool. LATE-PCR emphasises the importance of utilising a limiting primer with a higher melting temperature $\left(T_{m}\right)$ than that of the excess primer. We used primers incorporating a single base $(A-A)$ mismatch within
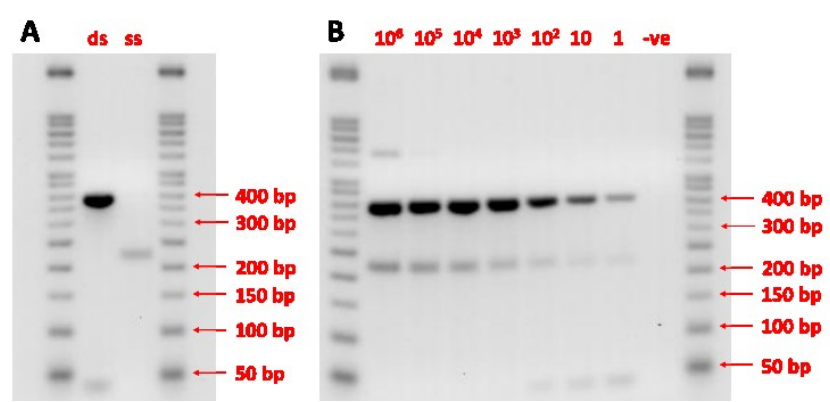

Figure 4. Gel electrophoresis: PCR amplicons compared against a 50 bp ladder in $2 \%$ agarose gel containing $1 x$ GelRed. A. Comparison of dsDNA PCR product and lambda exonuclease digested SSDNA PCR product amplified from $10^{4}$ copy numbers B. Asymmetric PCR amplification range from $10^{6}-1$ copy numbers with no template negative control.

the excess primer, lowering the $T_{m}$ below that of the limiting primer. A dsDNA product was amplified and digested into ssDNA using lambda exonuclease, providing a size reference for comparison to the asymmetric product. An excess: limiting primer concentration ratio of 10: 1 was optimal for ssDNA amplification. The asymmetric PCR product contained a band (above) that corresponded in size to the standard dsDNA amplification and a band (below) that corresponded to the ssDNA digest indicating successful one-pot amplification of SSDNA. A LATE-PCR limit of detection was demonstrated to single digit copy numbers (Figure 4).

Following asymmetric amplification, hybridisation was performed using the head-to-head orientation in 0.3 M PBS spiked with $5 \%$ PEG 10000. Initially, it was not possible to hybridise to the target using a constant temperature. This was attributed to secondary structure formation within the probe hybridisation region that contained the single nucleotide polymorphisms of interest for species-specificity. A heat-andcool approach was employed to melt secondary structures and allow probes to hybridise as the temperature was ramped down. Samples were heated to $95{ }^{\circ} \mathrm{C}$ for $3 \mathrm{~min}$ and gradually cooled to $55{ }^{\circ} \mathrm{C}$ over $1 \mathrm{~h}$. DMSO was added at $5 \%(\mathrm{v} / \mathrm{v})$ as an alternative to Tween 20. DMSO is a commonly utilised additive for the optimisation of PCR amplification and DNA sequencing, in circumstances for which GC-rich DNA regions would otherwise be prohibitive due to secondary structure formation. ${ }^{19}$, 20, 21, 22 This generated a quantitative volumetric range with asymmetric PCR product added in $10 \mu \mathrm{L}$ increments from $10 \mu \mathrm{L}$ to $50 \mu \mathrm{L}$ (Figure 5).

The signal intensity produced with the asymmetric PCR product was reduced as compared to the synthetic target with a 5: 1 on-to-off ratio achieved for the maximum volume of PCR product added $(50 \mu \mathrm{L})$. It is likely that the greater length of the PCR product (383 bases) as compared to the synthetic target (100 bases) could have influenced the hybridisation kinetics due to increased likelihood of secondary structure formation. Therefore, further investigation will be required to understand the effect of target secondary structure and apply SERS detection of asymmetric PCR product without the requirement for heating and cooling. 


\section{Conclusion}

A PCR-SERS coupling approach has been successfully demonstrated for the direct detection of a long PCR amplicon generated by the asymmetric PCR approach. This was founded

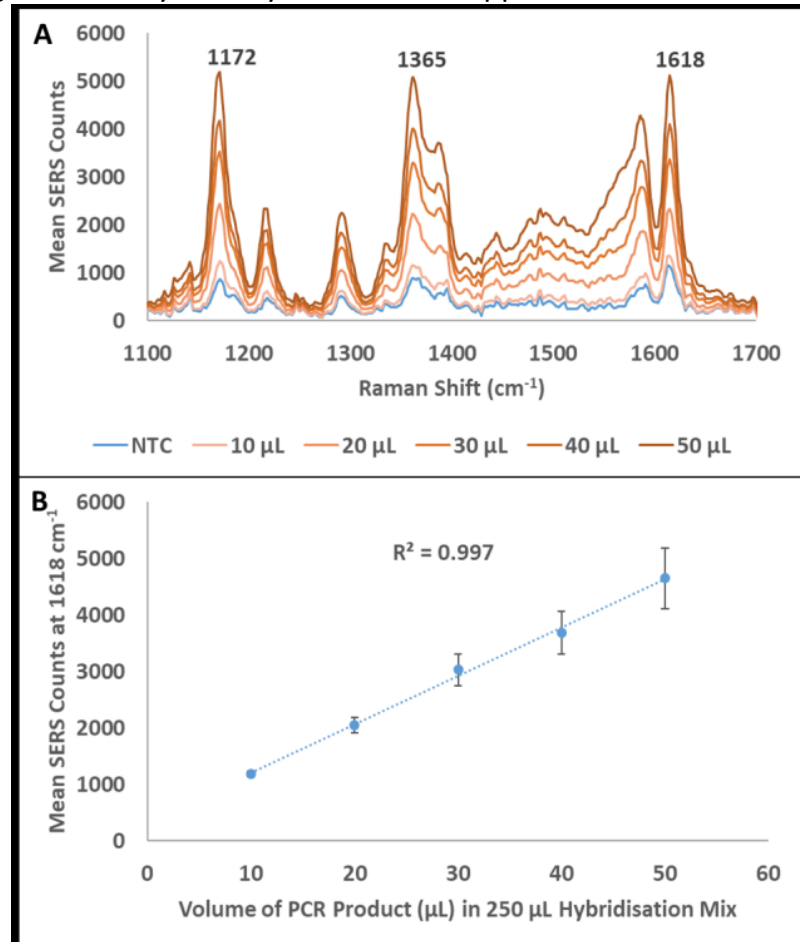

Figure 5. SERS analysis: Conjugates (10 pM) hybridised to volumetric range of asymmetric PCR product (amplified from $10^{4}$ copy numbers) in $0.3 \mathrm{M}$ PBS with $5 \%$ PEG 10000 and $5 \%$ DMSO upon cooling from $95-45^{\circ} \mathrm{C}$ in 1 hour. A. Mean ( $\left.N=3\right)$ SERS spectra (1 second accumulation time) following volumetric addition (10 $\mu \mathrm{L}$ incremental increases) of PCR product. B. Coefficient of determination $\left(R^{2}\right)$ value of 0.997 associated with volumetric range with error bars representative of standard deviation. Data accumulated using a $532 \mathrm{~nm}$ laser wavelength and $10 \%(5 \mathrm{~mW})$ laser power.

upon the initial optimisation of hybridisation conditions with use of an extended synthetic target, as much literature to date has emphasised the detection of short synthetic targets for proof-of-principle purposes, or biological targets such as microRNA sequences which are by nature, short and singlestranded in form and structure. This is likely due to difficulty in accomplishing hybridisation to long-chain DNA fragments using DNA-functionalised nanoparticles, for which we found the addition of high molecular weight polyethylene glycol (PEG) to be essential. The use of PEG 10000 was advantageous as compared to ionic aggregating agents, as sequence-specific assembly was maintained. Furthermore, PEG 10000 did not produce interfering background signal and provided a significant analytical time prior to loss of suspension within the assembled colloidal sample. Key findings include the necessity of using a high-molecular weight polymer to induce a hybridisation recognition event in the head-to-head orientation which provided the optimal SERS response. Additionally, asymmetric PCR was found to be a viable method for synthesising a ssDNA amplicon for targeting directly, thereby circumventing the necessity for further target separation steps. It should be noted that SERS enhancement was reduced for the longer PCR target (383 bases) as compared to the synthetic target (100 bases), although the complexity of potential target secondary structures and the necessity for a heat and cool hybridisation approach removed the equilibrium thermodynamics of nanoparticle assembly as applied in the first instance. Therefore, future investigation will emphasise understanding of long-chain ssDNA secondary structure and detection limitations for long-chain fragments beyond 100 nucleobases in length, with the aim of exploiting the full sensitivity potential of asymmetric PCR-SERS.

\section{Conflicts of interest}

There are no conflicts to declare.

\section{Acknowledgements}

The authors would like to thank Renishaw PIc and the EPSRC for the research funding provided. The research data associated with this paper will become available from the University of Strathclyde at the following link from (TBC) 2019: https://doi.org/10.15129/097e0cf0-615d-45c8-8c8fde8c8c82cab8.

\section{Notes and references}

S. Laing, K. Gracie and K. Faulds, Chem. Soc. Rev., 2016, 45, 1901-1918.

2 L. E. Jamieson, S. M. Asiala, K. Gracie, K. Faulds and D. Graham, Annu. Rev. Anal. Chem., 2017, 10, 415-437.

3 D. Graham, D. G. Thompson, W. E. Smith and K. Faulds, Nat. Nanotechnol., 2008, 3, 548-551.

4 D. Graham, B. J. Mallinder, D. Whitcombe, N. D. Watson and W. E. Smith, Anal. Chem., 2002, 74, 1069-1074. S. Hibbitts, P. L. White, J. Green, G. McNay, D. Graham and R. Stevenson, Anal. Methods, 2014, 6, 1288-1290. X. Li, T. Yang, C. S. Li, D. Wang, Y. Song and L. Jin, Sci. Rep., 2017, 7, 4771.

$7 \quad$ X. Li, T. Yang, C. S. Li, Y. Song, H. Lou, D. Guan and L. Jin, Theranostics, 2018, 8, 1678-1689.

$8 \quad$ X. Li, T. Yang, C. S. Li, L. Jin, H. Lou and Y. Song, Biomed. Opt. Express, 2018, 9, 3167.

9
E. J. H. Wee, Y. Wang, S. C.-H. Tsao and M. Trau, Theranostics, 2016, 6, 1506-1513.

10 S. Mabbott, D. Thompson, N. Sirimuthu, G. McNay, K. Faulds and D. Graham, Faraday Discuss., 2016, 187, 461472.

R. M. Amasino, Anal. Biochem., 1986, 152, 304-307. D. Miyoshi and N. Sugimoto, Biochimie, 2008, 90, 10401051.

13 S. Nakano, D. Miyoshi and N. Sugimoto, Chem. Rev., 2014, 114, 2733-2758.

14 L. S. Lerman, Proc. Natl. Acad. Sci., 1971, 68, 1886-1890.

15 V. V. Vasilevskaya, a. R. Khokhlov, Y. Matsuzawa and K. 
Yoshikawa, J. Chem. Phys., 1995, 102, 6595.

C. H. Spink and J. B. Chaires, J. Am. Chem. Soc., 1995, 117, 12887-12888.

17 J. A. Sanchez, K. E. Pierce, J. E. Rice and L. J. Wangh, Proc. Natl. Acad. Sci., 2004, 101, 1933-1938.

18 K. E. Pierce, J. A. Sanchez, J. E. Rice and L. J. Wangh, Proc. Natl. Acad. Sci., 2005, 102, 8609-8614.

19 P. R. Winship, Nucleic Acids Res., 1989, 17, 1266.

20 B. Bachmann, W. Lüke and G. Hunsmann, Nucleic Acids Res., 1990, 18, 1309.

21 K. Varadaraj and D. M. Skinner, Gene, 1994, 140, 1-5.

22 R. Chakrabarti and C. E. Schutt, Nucleic Acids Res., 2001, 29, 2377-2381.

23 N. Leopold and B. Lendl, J. Phys. Chem. B, 2003, 107, 57235727.

24 R. Owczarzy, A. V. Tataurov, Y. Wu, J. A. Manthey, K. A. McQuisten, H. G. Almabrazi, K. F. Pedersen, Y. Lin, J. Garretson, N. O. McEntaggart, C. A. Sailor, R. B. Dawson and A. S. Peek, Nucleic Acids Res., 2008, 36, W163-W169. D. Paramelle, A. Sadovoy, S. Gorelik, P. Free, J. Hobley and D. G. Fernig, Analyst, 2014, 139, 4855. X. Zhang, M. R. Servos and J. Liu, Chem. Commun., 2012, 48, 10114. F. Menges, Spectragryph - Optical Spectroscopy Software, http://www.effemm2.de/spectragryph/index.html. 